\title{
Seguridad ocupacional para mejorar el bienestar de los trabajadores en industrias alimentarias
}

\author{
Regner Nicolás Castillo Salazar ${ }^{1}$ \\ rcastillos@ucv.edu.pe \\ https://orcid.org/0000-0001-8956-2402 \\ Fernando Jesús Valencia Briceño ${ }^{2}$ \\ fjvalenciab@ucvvirtual.edu.pe \\ https://orcid.org/0000-0002-0711-6204
}

\section{RESUMEN}

La investigación realizada tuvo como objetivo principal desarrollar un sistema de seguridad ocupacional para mejorar el desempeño laboral de los trabajadores de la molinera Santa Mónica S.C.R.L en la ciudad de Moyobamba, 2020. El tipo de estudio realizado fue una investigación aplicada de tipo pre-experimental del cual se tuvo una muestra de 7 trabajadores de la empresa, utilizando la técnica de encuesta e instrumento de cuestionario, dándonos como resultado que el desempeño laboral de los trabajadores mejoró significativamente después de la implementación de seguridad ocupacional, de tal manera se rechazó la hipótesis nula y se aceptó la hipótesis general de la investigación. En los resultados obtenidos se pudo evidenciar que el nivel de seguridad antes de la implementación del sistema fue del 59\% en el indicador negativo, por otro lado, el nivel de desempeño fue del $63 \%$ en el nivel alto siendo un resultado muy positivo, luego los resultados pos test nos indicaron una mejora en el desempeño laboral al 76\% demostrando así resultados favorables en el sistema de seguridad ocupacional para la empresa.

Palabras clave: seguridad ocupacional; desempeño laboral; sistema; riesgo laboral

\footnotetext{
${ }^{1}$ Doctor en Gestión Pública y Gobernabilidad, Maestro en Gestión Pública, Licenciado en Administración, Jefe de Investigación Formativa y Docente, Universidad César Vallejo, Moyobamba, Perú.

${ }^{2}$ Bachiller de la Escuela Profesional de Administración, Universidad César Vallejo, Moyobamba, Perú.
} 


\title{
Occupational safety to improve the well-being of workers in food industries
}

\begin{abstract}
The main objective of the research was to develop an occupational safety system to improve the work performance of the workers of the Santa Monica Mill S.C.R.L in the city of Moyobamba, 2020. The type of study carried out was an applied research of pre-experimental type of which we had a sample of 7 workers of the company, using the technique of survey and instrument of questionnaire, giving us as result that the labor performance of the workers improved significantly after the implementation of occupational safety, in such a way the null hypothesis was rejected and the general hypothesis of the research was accepted. In the results obtained, it was possible to evidence that the safety level before the implementation of the system was $59 \%$ in the negative indicator, on the other hand, the performance level was $63 \%$ in the high level being a very positive result, then the post-test results indicated an improvement in the labor performance to $76 \%$ showing favorable results in the occupational safety system for the company.
\end{abstract}

Keywords: occupational safety; work performance; system; occupational risk

Artículo recibido: 02 Setiembre. 2021 Aceptado para publicación: 30 Setiembre. 2021

Correspondencia: rcastillos@ucv.edu.pe Conflictos de Interés: Ninguna que declarar 


\section{RESUMO}

O principal objectivo da investigação era desenvolver um sistema de segurança no trabalho para melhorar o desempenho laboral dos trabalhadores do moinho Santa Monica S.C.R.L na cidade de Moyobamba, em 2020. O tipo de estudo realizado foi uma pesquisa aplicada pré-experimental da qual tivemos uma amostra de 7 trabalhadores da empresa, utilizando a técnica de inquérito e instrumento de questionário, o que nos deu como resultado que o desempenho laboral dos trabalhadores melhorou significativamente após a implementação da segurança no trabalho, de tal forma que a hipótese nula foi rejeitada e a hipótese geral da pesquisa foi aceite. Nos resultados obtidos foi possível demonstrar que o nível de segurança antes da implementação do sistema foi de $59 \%$ no indicador negativo, por outro lado, o nível de desempenho foi de $63 \%$ no elevado nível sendo um resultado muito positivo, então os resultados pós-teste indicaram uma melhoria no desempenho do trabalho para $76 \%$ demonstrando resultados favoráveis no sistema de segurança no trabalho para a empresa.

Palavras-chave: segurança no trabalho, desempenho no trabalho, sistema, risco profissional 


\section{INTRODUCCIÓN}

La seguridad laboral se ocupa de la gestión o manejo de los riesgos inherentes a las operaciones, procedimientos, actividades comerciales y otros entornos; siendo un punto que ocupa múltiples campos como el esfuerzo, riesgos inminentes, estabilidad en el área de trabajo y todo lo que pueda corresponder al ambiente laboral, para que los colaboradores tengan bienestar físico, mental y social (Green, 2011). La prevención que deben tener ellos frente a los riesgos que existen y las consecuencias negativas en el área de su trabajo, pueden tener complicaciones en la salud, que hará que su desempeño sea bajo y no rindan como debieran (Vega-Monsalve, 2017).

En la actualidad el propósito de toda empresa debería consistir en un constante actualización e incrementación de las medidas de seguridad en los lugares de trabajo de todo el personal, por lo que se hace necesario poder establecer y actualizar las disposiciones del reglamento para que pueda garantizar un trabajo saludable y libre de riesgos. Las empresas privadas y públicas deben de realizar programas para conseguir ambientes saludables, y así prevenir las enfermedades y accidentes de trabajo (JaimesMorales, 2018).

Informarse, conocer tus derechos y tus responsabilidades. En todos los países y a nivel mundial existen leyes y reglas de Seguridad y Salud Laboral que establece las normas que han de seguir los empresarios, así como leyes de Prevención de Riesgos Laborales (LPRL) (Schulte et al., 2005). Estas leyes tienen como objetivo el desarrollo de las actividades necesarias para conocer de forma anticipada los riesgos que pueden existir en cualquier puesto de trabajo. Parte de la idea de que, si la tarea está bien estudiada de antemano, sabiendo cómo hay que hacerla y qué medios hay que emplear, también se podrán prever los riesgos que puedan aparecer (Pana-Cryan et al., 2018).

Conocer los procedimientos de seguridad necesarios nos ayudará a trabajar en un entorno más seguro y saludable ya que es muy importante estar informado de todos los cambios referentes a las regulaciones de seguridad y comprobar que sus políticas y procedimientos se actualicen y comuniquen correctamente a los empleados (Suharyo \& Bastari, 2020). Involucrar a los trabajadores en su propia seguridad favorecerá la creación de un ambiente laboral más tranquilo (Neuhaus et al., 2014).

Identificar posibles riesgos en tu puesto de trabajo, siendo importante identificar estos riesgos potenciales y poder solucionarlo de manera eficaz.(Feng \& Trinh, 2019) De la 
misma forma, confirma que conoces los procedimientos de reporte y comunicación ante posibles riesgos en el trabajo. Esto ayudará a identificar el problema y ponerlo en conocimiento del empresario, quien tiene como obligación solucionarlo y asegurar que el entorno de trabajo sea un lugar libre de peligros (Riaño-Casallas et al., 2016).

Proporcionar orientación laboral y capacitación para el trabajo, puesto que la mayoría de accidentes de trabajo se evidencian dentro de los primeros 6 meses, la formación de los trabajadores en salud y seguridad es esencial para certificar que alcancen los conocimientos adecuados a la hora de desempeñar su trabajo de una manera competente y segura (Cuesta, 2018).

Al contratar a un nuevo profesional, es importante ofrecerle la orientación especifica con el objetivo de prevenir posibles riesgos y accidentes durante el desempeño de su actividad profesional (Misiurek \& Misiurek, 2017).

Contar con el equipo de protección personal específico (EPP). El empresario tiene la obligación de proporcionar el equipamiento adecuado y de encargarse de que el trabajador reciba la formación requerida para el correcto uso de éste (Aburumman et al., 2019). Por otra parte, el trabajador tiene el derecho de exigir que se le proporcionen estas herramientas y la obligación de hacer un buen uso de las mismas (Fargnoli \& Lombardi, 2020).

Supervisar las labores de los trabajadores una de las maneras más efectivas para garantizar la seguridad de los trabajadores es mediante la instrucción y supervisión de sus labores (Marshall, 2020). Puesto que puede ser una tarea para un empleado con mayor experiencia y conocimientos sobre los procedimientos de seguridad (Nik Him et al., 2020).

Disponer de un entorno sano y seguro donde trabajar es, tal y como apuntamos al inicio del artículo, una responsabilidad compartida entre el empresario y el trabajador que beneficia a ambas partes en la confianza y un mejor entorno de calidad. La formación en seguridad es primordial para cambiar la tendencia de accidentes laborales que se ha ido registrando en los últimos años en nuestro país (Tamers et al., 2020).

Por otro lado la región de San Martín debatió sobre diferentes temas de seguridad ocupacional dentro de las empresas peruanas en donde se destacó que las empresas suelen invertir al desarrollo de algún sistema en relación cuando se involucra asuntos legales y pasaría de ser una opción a resultar siendo una obligación. 
Y siendo en la ciudad de Moyobamba nuestro punto de investigación como en cualquier otra ciudad en constante crecimiento con una población de aproximadamente de 60,000 ciudadanos presentan casos de intensidad baja a media de accidentes e incidentes laborales en las empresas, donde en la mayor tasa de casos se evidencian en las empresas e industrias de procesamiento, la gran mayoría de estas no presentan un sistema de seguridad ocupacional como es en la Molinera Santa Mónica, donde lleva ejerciendo desde la década de los 80 's, a lo largo de los años ha habido accidentes laborales en donde no se ha tomado su respectiva importancia al caso dando como resultado un impacto negativo en el desempeño de los colaboradores como en la economía de la industria.

\section{MARCO TEÓRICO}

Basándose en la revista científica: Workplace health and safety. (2019), (Artículo científico). La empresa internacional Nestlé nos enseña que la cultura en el trabajo influye en la salud y el bienestar de los colaboradores. Un ambiente positivo, en el que los empleados sienten que son tratados con respeto y justicia y donde cuentan con el apoyo de los líderes, puede influir mucho en el compromiso y la productividad. Más allá de eso, también evaluamos el presentismo: pérdida de productividad en el trabajo representada por limitaciones de problemas de salud que interfieren con aspectos específicos del trabajo, como el rendimiento, que tiene un efecto aún más perjudicial en la productividad desempeñándose en niveles más bajos. En 2019 se realizaron una serie de seminarios web dirigidos por expertos, que enriquecieron el conocimiento del grupo sobre la salud, se realizaron 22 seminarios web que involucraron a 594 participantes e incluyeron temas como estrategia de salud, KYNP, \#HealthyLives, informes de enfermedades y estrés y resistencia, por otro lado el programa utilizado, Know Your Numbers ( KYNP) está diseñado para permitir que los empleados comprendan sus riesgos de salud y nos permite medir los riesgos de salud clave de nuestra población de empleados, lo que nos ayuda a comprender mejor el perfil de salud de nuestros empleados y el impacto de la salud en la productividad, y para construir intervenciones estratégicas, focalizadas y basadas en evidencia. Esto es fundamental para construir una organización sostenible y ofrecer un rendimiento de mercado superior.

A su vez Nadhim, Hon, y Stewart, (2018), Investigating the Relationships between Safety Climate and Safety Performance Indicators in Retrofitting Works, (Artículo científico), Universidad Tecnológica de Queensland, Australia. En conclusión, del artículo no indica 
mejorar la sostenibilidad de los edificios existentes y/o antiguos mediante el empleo de obras de remodelación, renovación, renovación y remodelación ya que se ha vuelto muy demandado en las últimas décadas a nivel mundial, esta necesidad ha planteado las preocupaciones de las normas de seguridad en trabajos de retroacción que normalmente son proyectos pequeños por esto dentro de la investigación se rescató una contribución que fue la determinación de factores efectivos del clima de seguridad. Al emplear el cuestionario NOSACQ-50, esta investigación ha ofrecido una evaluación de seguridad inicial en lugar de confiar en las estadísticas simples de la industria de la construcción, esto conllevó a que más de la mitad de los factores del clima de seguridad se implementaron recientemente en trabajos de retroalimentación, como el empoderamiento y la justicia de la seguridad de la gestión así como la comunicación y el aprendizaje de los trabajadores y por último asegurar la eficacia de los sistemas y así la confianza de los colaboradores con los cambios propuestos.

Por consiguiente, Druley, (2018), The Hierarchy of Controls. (artículo científico). Nos define 5 peldaños de los cuales organiza de manera efectiva el control y prevención de riesgos en un mayor intento de suprimir cualquier amenaza, los cuales son, eliminación física del peligro; sustitución o reemplazo del peligro; control de la ingeniería aislando a las personas del peligro; controles administrativos respecto al cambio en la forma de trabajar de las personas y por último la protección del trabajador con EPP. Identificar y mitigar las exposiciones a riesgos laborales antes de que comience el trabajo es el objetivo de toda la organización y sus componentes: "No se pueden eliminar todos los peligros, pero cuanto más se acerque a la cima, más cerca podrá alcanzar ese ideal y hacer que las personas estén más saludables y seguras" (Jonathan Bach, 2016, p.2)

Para Smith, (2018). Workplace Safety: Culture, Plans, and Actions. (Artículo científico). Nos enseña que dentro de la seguridad en el trabajo debe existir una fuerte cultura de seguridad, de valores y creencias que los empleadores y empleados deben compartir en relación con los riesgos en el lugar de trabajo por esto el liderazgo es crítico en el cambio cultural ya que entablar un fuerte ambiente de seguridad es complejo y difícil en muchos aspectos. Los líderes deben adoptar la agenda de seguridad en toda la empresa como también guiar los esfuerzos en el cambio dentro de toda la organización.

Como también Shaheen, Sami, Fais, Abdul y Gamal, (2017), Employee job security and performance relationship in developing economy through employee engagement: Critical 
analysis with PLS-SEM, (artículo científico), Revista Internacional de Investigación de Calidad, Bangladesh. Nos explica que la organización y los trabajadores esperan llevar su labor con normalidad y seguridad siento esto un punto demandante en las acciones a tomar sobre la seguridad en el trabajo. La seguridad laboral de los trabajadores se considera un elemento vital en la organización para aumentar su desempeño laboral, lo que en última instancia fomenta la productividad total de la organización, además, hace algunas décadas, se han realizado varios estudios y se han encontrado resultados positivos en la relación de seguridad y desempeño laboral por este motivo es que los empleados no dudan en trabajar duro para cumplir los objetivos de la organización cuando se les confía que su área laboral cumple con las medidas de seguridad adecuadas.

\section{OBJETIVOS}

Se propone como objetivo general identificar cómo la implementación del sistema de seguridad ocupacional incrementa el nivel de desempeño laboral de los trabajadores de la molinera Santa Mónica, periodo 2020. Objetivos específicos, Definir el nivel de seguridad ocupacional de los trabajadores de la Molinera Santa Mónica, periodo 2020. Definir el nivel de desempeño laboral de los trabajadores de la Molinera Santa Mónica, periodo 2020. Diseñar un sistema de seguridad ocupacional para la empresa Molinera Santa Mónica, periodo 2020. Evaluar el nivel de desempeño laboral de los trabajadores después de haber implementado el sistema en la Molinera Santa Mónica, periodo 2020.

\section{METODOLOGÍA}

La presente investigación fue de tipo aplicada, de diseño pre experimental en donde la población de estudio estuvo constituida por 7 trabajadores de género masculino de la empresa en donde se incluyeron el área de gerencia como de producción y se excluyeron al área de contabilidad al tratarse de un apoyo externo. La muestra fue igual que la población al tratarse de solo 7 personas habiendo utilizado el muestreo no probabilístico por conveniencia.

El análisis del sistema de seguridad ocupacional se dimensionó en: Gestión de seguridad y Gestión de salud, los cuales fueron medidas en una escala de forma ordinal.

El análisis del desempeño laboral se dimensiona en: Eficiencia y eficacia las cuales serán medidas en una escala de forma ordinal.

La técnica utilizada para llevar a cabo el estudio con el fin de recopilar datos e información sobre la empresa fue la encuesta y el instrumento de recolección de datos fue 
el cuestionario el cual se estructuró en 34 ítems en total, 16 preguntas dentro de la variable Seguridad ocupacional y 18 preguntas dentro de la variable Desempeño laboral como también contamos con 4 dimensiones con su respectivos indicadores, en nuestra primera variable $(\mathrm{X})$ tenemos 2 dimensiones con 15 indicadores y en nuestra segunda variable (Y) tenemos 2 dimensiones con 7 indicadores.

Para ejecutar la investigación se realizó el siguiente procedimiento, se llevó a cabo las encuestas a los colaboradores de la Molinera para así recopilar información según la situación vivencial de cada uno y a su vez se respondieron las preguntas según la guía de observación para tener una propia evaluación tanto del ambiente laboral como de las experiencias vistas por los gerentes generales y así tener una noción general de la problemática para diseñar con precisión el sistema de seguridad ocupacional y así determinar el mejoramiento del desempeño laboral en un pre y post test siendo estos datos necesarios para la comparación de estos dos aspectos.

El desarrollo de los datos recolectados mediante los instrumentos utilizados en el proyecto de investigación tienen que explicarse mediante un análisis específico para así lograr los objetivos propuestos, procesados por el formato de Excel, una herramienta funcional que permitió representar de manera clara datos precisos y determinantes, de tal manera se pudo interpretarlos y así obtener resultados que permitieron dar solución a la problemática y con ello se diseñó de manera efectiva el sistema de seguridad ocupacional siguiendo los pasos a continuación:

- Edición de datos: En primer lugar, se digitó los datos obtenidos de la investigación para tener un orden y correcta relación entre ambas variables.

- Ordenamiento: Luego se pasó a ordenar los datos según el nombre, tipo, etiqueta y valores correspondientes.

- Tabulación: El formato nos dio resultados que fueron utilizados para determinar y diseñar los cuadros estadísticos.

- Análisis e interpretación de datos: Para concluir se compararon los resultados con las variables propuestas y sus respectivas hipótesis determinando así la correlación adecuada en las características y así se determinó una solución a la problemática como a su vez sus respectivas conclusiones y sugerencias. 


\section{RESULTADOS}

A continuación, se presentan los resultados evidenciados del proceso de investigación, así como el cumplimiento de objetivos y la verificación de las hipótesis.

\section{Cumplimiento de objetivos específicos.}

1. Definir el nivel de seguridad ocupacional de los trabajadores de la Molinera Santa Mónica, periodo 2020.

Tabla $\mathbf{N}^{\circ}$ 01. Nivel de seguridad ocupacional - Pre test

\begin{tabular}{lcccccc}
\hline \multicolumn{1}{c}{ Nivel } & \multicolumn{2}{c}{ Seguridad } & \multicolumn{2}{c}{ Salud } & \multicolumn{2}{c}{ Total } \\
& F & $\mathbf{\%}$ & F & \% & F & \% \\
\hline Bajo & 4 & $57 \%$ & 4 & $57 \%$ & 3 & $43 \%$ \\
Medio & 2 & $29 \%$ & 1 & $14 \%$ & 2 & $29 \%$ \\
Alto & 1 & $14 \%$ & 2 & $29 \%$ & 2 & $29 \%$ \\
Total: & $\mathbf{7}$ & $\mathbf{1 0 0 \%}$ & $\mathbf{7}$ & $\mathbf{1 0 0 \%}$ & $\mathbf{7}$ & $\mathbf{1 0 0 \%}$ \\
\hline
\end{tabular}

Fuente: Encuestas realizadas a los trabajadores de la molinera Santa Mónica

S.C.R.L.

\section{Interpretación}

Se representa el nivel de seguridad ocupacional (pre test) de la molinera Santa Mónica S.C.R.L. según los resultados en a nivel general se percibió un $43 \%$ en el nivel bajo. Respecto a los ítems de seguridad y salud se obtuvo en el nivel bajo un 57\%, representando un claro resultado negativo para la variable de seguridad ocupacional, de acuerdo al mayor porcentaje en el nivel alto se obtuvo en el indicador salud con $29 \%$.

Por lo tanto, se acepta la primera hipótesis específica de la investigación.

\section{Definir el nivel de Desempeño laboral de los trabajadores de la Molinera Santa} Mónica, periodo 2020.

Tabla $\mathbf{N}^{\circ}$ 02. Nivel de desempeño laboral - Pre test

\begin{tabular}{lcccccc}
\hline \multicolumn{1}{c}{ Nivel } & \multicolumn{2}{c}{ Eficiencia } & \multicolumn{2}{c}{ Eficacia } & \multicolumn{2}{c}{ Total } \\
& F & \% & F & \% & F & \% \\
\hline Bajo & 1 & $14 \%$ & 0 & $0 \%$ & 1 & $14 \%$ \\
Medio & 2 & $29 \%$ & 1 & $14 \%$ & 2 & $29 \%$ \\
Alto & 4 & $57 \%$ & 6 & $86 \%$ & 4 & $57 \%$ \\
Total: & 7 & $100 \%$ & 7 & $100 \%$ & 7 & $100 \%$ \\
\hline
\end{tabular}

Fuente: Encuestas realizadas a los trabajadores de la molinera Santa Mónica S.C.R.L. 


\section{Interpretación}

Se representa el nivel de desempeño laboral (pre test) de la molinera Santa Mónica S.C.R.L. según los resultados a nivel general se percibió un 57\% en el nivel alto. Respecto al ítem de eficacia se obtuvo en el nivel alto un $86 \%$, representando un claro nivel alto de la variable de desempeño laboral, de acuerdo al mayor porcentaje en el nivel bajo con $0 \%$ se obtuvo del ítem de eficacia.

\section{3. diseñar un sistema de seguridad ocupacional para la empresa Molinera Santa}

\section{Mónica, periodo 2020.}

El modelo de sistema de seguridad ocupacional se ha realizado de acuerdo a los resultados obtenidos de las encuestas y las observaciones en la empresa, se ha tratado de ser conciso en los procedimientos y evaluación de riesgos para minimizar las problemáticas encontradas en el procedimiento de investigación como también la obtención de EPP de manera que se pueda aprovechar los equipos y que no represente una gran inversión para la molinera Santa Mónica S.C.R.L.

Según la tercera hipótesis se puede aceptar de manera efectiva por la implementación del sistema de seguridad a la empresa y de manera que resulta favorable en el mejoramiento de las variables antes y después de los test.

4. Evaluar el nivel de desempeño laboral de los trabajadores después de haber implementado el sistema en la Molinera Santa Mónica, periodo 2020.

Tabla $\mathbf{N}^{\circ} \mathbf{0 3}$. Nivel de desempeño laboral - Post test

\begin{tabular}{lcccccc}
\hline \multicolumn{1}{c}{ Nivel } & \multicolumn{2}{c}{ Eficiencia } & \multicolumn{2}{c}{ Eficacia } & \multicolumn{2}{c}{ Total } \\
\hline Bajo & F & \% & F & \% & F & $\%$ \\
Medio & 1 & $14 \%$ & 0 & $0 \%$ & 1 & $14 \%$ \\
Alto & 2 & $29 \%$ & 1 & $14 \%$ & 1 & $14 \%$ \\
Total: & 4 & $57 \%$ & 6 & $86 \%$ & 5 & $71 \%$ \\
\hline
\end{tabular}

Fuente: Encuestas realizadas a los trabajadores de la molinera Santa Mónica S.C.R.L. Interpretación

Se puede observar que el nivel de desempeño laboral (post test) de la molinera Santa Mónica S.C.R.L. según los resultados a nivel general se percibió un incremento al $71 \%$ en el nivel alto, en el nivel medio se pudo percibir una reducción al 14\% y en el nivel bajo no hubo variación con un $14 \%$. 
Respecto a las dimensiones, la eficacia es el ítem con mayor porcentaje en el nivel alto con un $86 \%$, como también lleva el porcentaje más crítico del mismo ítem con un porcentaje del $0 \%$ en el nivel bajo.

De tal modo, la cuarta hipótesis específica de la investigación se estaría aceptando.

\section{Cumplimiento del objetivo general}

Cómo la implementación del sistema de seguridad ocupacional incrementa el nivel de desempeño laboral de los trabajadores de la molinera Santa Mónica, periodo 2020.

\section{Prueba de normalidad}

$\mathrm{P}>0.05$ los datos son normales

$\mathrm{P}<0.05$ no existe normalidad en los datos

Tabla $\mathrm{N}^{\circ}$ 04. Pruebas de normalidad del pre y post test.

\begin{tabular}{lcccccc}
\hline Test & \multicolumn{2}{c}{ Kolmogorov-Smirnov } & \multicolumn{3}{c}{ Shapiro-Wilk } \\
& Estadístico & gl & Sig. & Estadístico & gl & Sig. \\
Diferencia &, 190 & 7 &, $200^{*}$ &, 940 & 7 &, 635 \\
\hline
\end{tabular}

a. Corrección de significación de Lilliefors

\section{Interpretación}

Respecto a las pruebas de normalidad del nivel de desempeño laboral entre el pre y post test, así como su diferencia, se tomaron los resultados de la prueba de Shapiro - Wilk ya que tiene un mejor análisis y resultado para las muestras menores a 30, en esta investigación la muestra fue de 7 encuestados.

La prueba contrasta la siguiente hipótesis nula: Las variables evaluadas mantienen una distribución normal, siempre y cuando el nivel de significancia nos demuestre que siendo un valor mayor a 0.05 resulta aceptable, pero si resulta un valor menor, se rechaza. En este caso el análisis resultó un valor mayor a 0.05 , de tal manera se concluye que todas las variables siguen una distribución normal, por lo tanto, la hipótesis es aceptada.

\section{Prueba de Hipótesis}

$\mathrm{P}<0.05$ se acepta la hipótesis de la investigación

$\mathrm{P}>0.05$ Se rechaza la hipótesis de la investigación y se acepta la nula.

Hi: La implementación del sistema de seguridad ocupacional mejora significativamente el desempeño laboral de los trabajadores de la molinera Santa Mónica, periodo 2020. 
Ho: La implementación del sistema de seguridad ocupacional no mejorará significativamente el desempeño laboral de los trabajadores de la molinera Santa Mónica, periodo 2020

\section{Rangos con signos de Wilcoxon para desempeño laboral}

\begin{tabular}{llccc}
\hline \multicolumn{1}{c}{ Diferencia } & \multicolumn{1}{c}{ Rangos } & N & $\begin{array}{c}\text { Rango } \\
\text { promedio }\end{array}$ & $\begin{array}{c}\text { Suma de } \\
\text { rangos }\end{array}$ \\
\hline \multirow{2}{*}{ Post test -} & Rangos negativos & 2 & 4,50 & 9,00 \\
Pre test & Rangos positivos & 7 & 5,14 & 36,00 \\
& Empates & 1 & & \\
& Total & 10 & & \\
\hline
\end{tabular}

a. Postest $<$ Pretes

b. Postest > Pretes

c. Postest $=$ Pretes

\section{Interpretación}

En la tabla nos muestra que, respecto a la variable de desempeño laboral, 2 de los encuestados tienen mayor percepción por el pre test a comparación del post test, 7 encuestados han tenido mayor percepción del desempeño laboral logrado en el post test que en el pre test y, por último, 1 encuestado ha mantenido su percepción respecto al desempeño laboral entre los dos test.

Prueba de muestras emparejadas

\begin{tabular}{|c|c|c|c|c|c|c|c|}
\hline \multirow[t]{2}{*}{ Media } & \multirow[t]{2}{*}{$\begin{array}{l}\text { Desv. } \\
\text { Desviación }\end{array}$} & \multirow{2}{*}{$\begin{array}{l}\text { Desv. } \\
\text { Error } \\
\text { promedio }\end{array}$} & \multicolumn{5}{|c|}{$\begin{array}{l}\text { 95\% de intervalo de confianza de la } \\
\text { diferencia }\end{array}$} \\
\hline & & & Inferior & Superior & $\mathbf{t}$ & gl & $\begin{array}{l}\text { Sig. } \\
\text { (Bi) }\end{array}$ \\
\hline $\begin{array}{ll}\text { Pre test } & --585,714 \\
\text { Post test } & \end{array}$ & 401,782 & 151,859 & $-957,300$ & $-214,128$ & $-3,857$ & 6 & ,008 \\
\hline
\end{tabular}

\section{Interpretación}

Se puede observar de los resultados obtenidos en la comparación de muestras relacionadas un valor de $t$ de $-3.85,6$ grados de libertad y Significancia (bilateral) de 0.008 siendo menos que 0.05 , por lo que el nivel de desempeño laboral es diferente entre el post y pre test. 


\section{DISCUSIÓN}

Después de haber logrado resultados concretos en el proceso de investigación por la implementación del sistema de seguridad en la molinera Santa Mónica S.C.R.L con el objetivo principal de mejorar el desempeño laboral se puede concluir que el efecto fue favorable y que tuvo significancia en los resultados finales ya que en el rango general en el nivel alto se obtuvo un aumento desde el 57\% al 71\%, dándonos como conclusión del caso que se obtuvo un aumento considerable del $14 \%$ entre el pre y post test, por otro lado, dentro del nivel medio se obtuvo un aumento del $29 \%$ al $14 \%$ explicándose que parte de los que consideraban un nivel medio en el pre test ahora consideran en el post test un nivel alto siendo resultados favorables para la investigación ya que en el nivel bajo se mantiene en el rango general un $14 \%$ y esto explicaría los datos existentes. Mencionando a los autores Koontz, H., Weihrich, H., y Cannice M. parte de los antecedentes que nos hablan de factores y puntos resaltantes en relación al desempeño laboral en una investigación del 2012 nos explican que la productividad se basa en el constante control de la efectividad y eficiencia tanto en aspecto individual como aspecto organizacional, de tal modo se define la efectividad como el cumplimiento de los objetivos propuestos y eficiencia es lograrlo con un mínimo de recursos. Sabiendo esto estas dos variantes suponen un apoyo mutuo en el desenvolvimiento empresarial en sus actividades y correcto cumplimiento de objetivos sin embargo por separados se tendrían resultados poco favorables al suponer que uno es más importante que el otro en tal caso que muchas empresas se vienen abajo por la mala organización en el desarrollo de sus actividades omitiendo la eficiencia de trabajar en conjunto. (p.14-15).

Respecto a la variable de seguridad ocupacional se obtuvieron resultados negativos ya que en las dimensiones tanto de seguridad como de salud se obtuvo en el nivel bajo un resultado del 57\%, esto representó que las condiciones de trabajo dentro de los ambientes laborables eran considerados moderadamente deficientes, respecto al nivel alto en la dimensión de seguridad se obtuvo solamente un resultado del $14 \%$ que consideran que las condiciones eran favorables y en el nivel intermedio solo un $29 \%$ en el resultado general, justamente por esto es que planteó la propuesta del sistema de seguridad ocupacional, para prevenir futuros accidentes laborables, evitar y en todo caso, eliminar cualquier riesgo inminente que pueda afectar a la integridad del trabajador así como de la empresa. Dentro de los antecedentes de la investigación podemos mencionar a Gutiérrez, 
(2019) en su investigación nos evidencia que hay mucha relación entre la seguridad industrial y el desempeño laboral ya que permite a los trabajadores desempeñarse de una mejor manera en sus áreas laborales al tener una mayor seguridad en sus procesos y ejecución en sus tareas diarias. También es importante que cada trabajador tenga sus EPP de acuerdo a la tarea para que puedan realizar sus deberes de manera segura, como también tener planes de motivación e instrucción de las normativas de seguridad para así tener un mayor control y conocimiento de la manera adecuada en el desenvolvimiento de sus actividades, información bastante relevante en relación a las condiciones de la molinera Santa Mónica S.C.R.L. así como también una guía para el mejoramiento y desarrollo de los puntos negativos que se pudieron evidenciar en la presente investigación.

\section{CONCLUSIONES}

Primera: El nivel de seguridad ocupacional antes de haber implementado el sistema de seguridad ocupacional en la molinera Santa Mónica S.C.R.L es baja, con un resultado del $59 \%$ demostrando que más de la mitad de trabajadores no están a gusto respecto a su seguridad dentro de sus ámbitos laborales.

Segunda: El nivel de desempeño laboral antes de haber implementado el sistema de seguridad ocupacional en la molinera Santa Mónica S.C.R.L es moderadamente alta, con un resultado del 63\%, esto nos indica que los trabajadores ejercen un nivel de desempeño considerable sin embargo aún hay aspectos que se deben mejorar para alcanzar un nivel mayor.

Tercera: Después de haber aplicado el sistema de seguridad ocupacional en la molinera Santa Mónica S.C.R.L con los parámetros establecidos resultó siendo adecuado y beneficioso para el desempeño laboral de los trabajadores ya que los resultados de la investigación arrojaron una diferencia de porcentajes positivos.

Cuarto: El nivel de desempeño laboral después de haber implementado el sistema de seguridad ocupacional en la molinera Santa Mónica S.C.R.L es alta, con un resultado del $76 \%$ demostrando así un aumento del $13 \%$ con resultados favorables tanto para el entorno del trabajador como para un mejor desenvolvimiento laboral.

\section{AGRADECIMIENTO}

En principio mi mayor agradecimiento a la empresa molinera Santa Mónica S.C.R.L por el apoyo con la obtención de información para el desarrollo de la investigación y a su vez 
por haberme permitido poder crear el sistema de seguridad del cual se pudo hacer efectiva con resultados favorables para la empresa.

En agradecimiento a la universidad Cesar Vallejo por brindarme los estudios y guías necesarias para poder efectuar de manera adecuada el informe de investigación, también, a los profesores por su apoyo en cada paso de esta investigación, su comprensión en los aspectos complicados y su gran labor como docentes profesionales en el camino académico que conllevó a culminar exitosamente esta etapa de mayor importancia en la vida profesional de uno como estudiante.

\section{REFERENCIAS}

Aburumman, M., Newnam, S., \& Fildes, B. (2019). Evaluating the effectiveness of workplace interventions in improving safety culture: A systematic review. Safety Science, 115, 376-392. https://doi.org/10.1016/j.ssci.2019.02.027

Cuesta Tamayo, K. D. (2018). Diseño de un modelo de gestión de seguridad y salud en el trabajo. Contexto, 7, 38-46. https://doi.org/10.18634/ctxj.7v.0i.837

Díaz, M. Seguridad en el trabajo y desempeño laboral. http://recursosbiblio.url.edu.gt/tesisjcem/2017/05/43/Diaz-Marleny.pdf

Druley, K. (2018). The Hierarchy of Controls. (artículo científico). https://www.safetyandhealthmagazine.com/articles/16790-the-hierarchy-ofcontrols

Fargnoli, M., \& Lombardi, M. (2020). Building Information Modelling (BIM) to Enhance Occupational Safety in Construction Activities: Research Trends Emerging from One Decade of Studies. Buildings, 10(6), 98. https://doi.org/10.3390/buildings10060098

Feng, Y., \& Trinh, M. T. (2019). Developing Resilient Safety Culture for Construction Projects. Journal of Construction Engineering and Management, 145(11), 04019069. https://doi.org/10.1061/(asce)co.1943-7862.0001720

Green, F. (2011). Unpacking the misery multiplier: How employability modifies the impacts of unemployment and job insecurity on life satisfaction and mental health. Journal of Health Economics, 30(2), 265-276. https://doi.org/10.1016/j.jhealeco.2010.12.005

Jaimes-Morales, J. (2018). Sistema de gestión de seguridad y salud en el trabajo: una revisión desde los planes de emergencia. IPSA Scientia, Revista Científica 
Multidisciplinaria, 3(1), 23-29. https://doi.org/10.25214/27114406.920

Marshall, T. M. (2020). Risk perception and safety culture: Tools for improving the implementation of disaster risk reduction strategies. International Journal of Disaster Risk Reduction, 47, 101557. https://doi.org/10.1016/j.ijdrr.2020.101557

Misiurek, K., \& Misiurek, B. (2017). Methodology of improving occupational safety in the construction industry on the basis of the TWI program. Safety Science, 92, 225-231. https://doi.org/10.1016/j.ssci.2016.10.017

Nadhim, E. Hon, Carol. Stewart, Ian. (2018). Investigating the Relationships between Safety Climate and Safety Performance Indicators in Retrofitting Works. Queensland University of Technology, Australia. (Artículo científico) https://www.researchgate.net/publication/326018608_Investigating_the_Relatio nships_between_Safety_Climate_and_Safety_Performance_Indicators_in_Retro fitting_Works

Neuhaus, M., Eakin, E. G., Straker, L., Owen, N., Dunstan, D. W., Reid, N., \& Healy, G. N. (2014). Reducing occupational sedentary time: a systematic review and metaanalysis of evidence on activity-permissive workstations. Obesity Reviews, 15(10), 822-838. https://doi.org/10.1111/obr.12201

Nik Him, N. F., Noor Aina Amirah, \& Hassan, A. (2020). exploratory factor analysis of safety management commitment and safety administration in the context construction industries. Asian People Journal (APJ), 3(1), 132-140. https://doi.org/10.37231/apj.2020.3.1.131

Pana-Cryan, R., Swanson, N., Chosewood, C., Reeves, K., Novicki, E., \& Atlanta, G. (2018). Healthy Work Design and Well-Being Program. https://doi.org/10.26616/NIOSHPUB2018152

Riaño-Casallas, M. I., Hoyos Navarrete, E., \& Valero Pacheco, I. (2016). Evolución de un sistema de gestión de seguridad y salud en el trabajo e impacto en la accidentalidad laboral: Estudio de caso en empresas del sector petroquímico en Colombia. Ciencia \& Trabajo, 18(55), 68-72. https://doi.org/10.4067/S071824492016000100011

Shaheen, A., Sami. M., Fais, A., Abdul, J. y Gamal, A. (2017). Employee job security and performance relationship in developing economy through employee engagement: Critical analysis with PLS-SEM, (artículo científico), International 
Journal for Quality Research (Bangladesh) https://www.researchgate.net/publication/322736951_Employee_job_security_a nd_performance_relationship_in_developing_economy_through_employee_eng agement_Critical_analysis_with_PLS-SEM

Schulte, P. A., Stephenson, C. M., Okun, A. H., Palassis, J., \& Biddle, E. (2005). Integrating Occupational Safety and Health Information Into Vocational and Technical Education and Other Workforce Preparation Programs. American Journal of Public Health, 95(3), 404-411. https://doi.org/10.2105/AJPH.2004.047241

Smith, J. (2018). Workplace Safety: Culture, Plans, and Actions. (Artículo científico). https://ohsonline.com/Articles/2018/09/01/Workplace-Safety-Culture-Plans-andActions.aspx?Page $=1$

Suharyo, O. S., \& Bastari, A. (2020). Analysis of The Implementation of Occupational Safety Program Efforts to Improve Work Productivity with Fault Tree Analysis Approach. 628-633.

Tamers, S. L., Streit, J., Pana-Cryan, R., Ray, T., Syron, L., Flynn, M. A., Castillo, D., Roth, G., Geraci, C., Guerin, R., Schulte, P., Henn, S., Chang, C., Felknor, S., \& Howard, J. (2020). Envisioning the future of work to safeguard the safety, health, and well-being of the workforce: A perspective from the CDC's National Institute for Occupational Safety and Health. American Journal of Industrial Medicine, 63(12), 1065-1084. https://doi.org/10.1002/ajim.23183

Vega-Monsalve, N. D. C. (2017). Nivel de implementación del Programa de Seguridad y Salud en el Trabajo en empresas de Colombia del territorio Antioqueño. Cadernos de Saúde Pública, 33(6). https://doi.org/10.1590/0102-311x00062516 